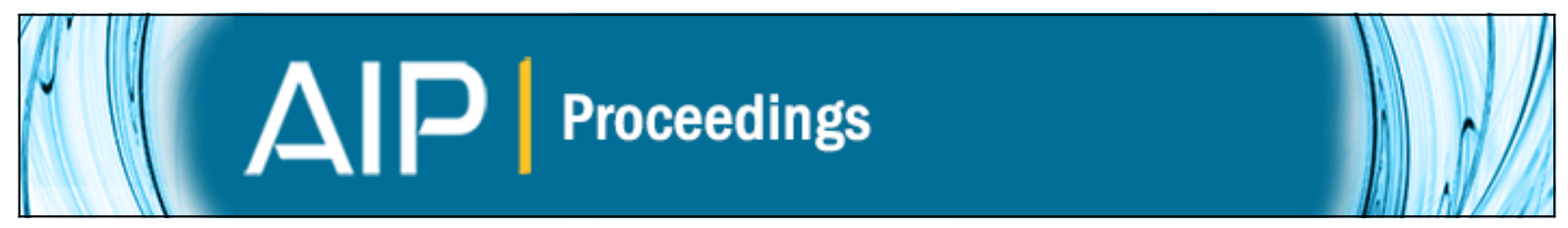

\title{
Higher fullerenes: Compositional analysis by EDXD and molecular dynamics
}

Marilena Carbone and Lorenzo Gontrani

Citation: AIP Conference Proceedings 1603, 40 (2014); doi: 10.1063/1.4883040

View online: http://dx.doi.org/10.1063/1.4883040

View Table of Contents: http://scitation.aip.org/content/aip/proceeding/aipcp/1603?ver=pdfcov

Published by the AIP Publishing

Articles you may be interested in

Molecular dynamics study of self-agglomeration of charged fullerenes in solvents

J. Chem. Phys. 138, 044318 (2013); 10.1063/1.4789304

Temperature influence on the crystallization of polyethylene/fullerene nanocomposites: Molecular dynamics simulation

J. Chem. Phys. 131, 234906 (2009); 10.1063/1.3275003

Molecular dynamics simulations on crystallization of polyethylene/fullerene nanocomposites

J. Chem. Phys. 130, 074902 (2009); 10.1063/1.3077864

Electronic states and luminescence in higher fullerene/porous Si nanocrystal composites

J. Chem. Phys. 124, 214706 (2006); 10.1063/1.2202742

Anticipatory programming —higher-order dynamics, fault-tolerance, composition

AIP Conf. Proc. 437, 507 (1998); 10.1063/1.56339 


\title{
Higher Fullerenes: Compositional Analysis by EDXD and Molecular Dynamics
}

\author{
Marilena Carbone ${ }^{1}$ and Lorenzo Gontrani ${ }^{2,3, a)}$ \\ ${ }^{1}$ Università di Roma Tor Vergata, Via della Ricerca Scientifica 1 I-00133 Roma, Italy \\ ${ }^{2}$ CNR-Istituto di Struttura della Materia, Via del Fosso del Cavaliere, I-00133 Roma, Italy \\ ${ }^{3}$ Dipartimento di Chimica- Università Sapienza, P.le Aldo Moro 5, I-00184 Roma, Italy \\ a) Corresponding author: lorenzo.gontrani@uniroma1.it
}

\begin{abstract}
A carbon soot of higher fullerenes was studied by Energy Dispersive X-ray Diffraction (EDXD) aiming at defining the overall structure as accurately as possible to define the overall structure, without lengthy separation and purification procedures. EDXD pattern was compared with model curves obtained for single homogeneous fullerenes already crystallized and for the hypothesized C180. All fullerenes contribute to the overall experimental curve to different extents, with C96 giving the best agreement. The presence of even higher fullerenes is very likely, since the hypothesized C180 gives a very good match as well.
\end{abstract}

Keywords: Higher Fullerenes, EDXD, Diffraction

PACS: 61,82

\section{INTRODUCTION}

Since their first discovery in 1985, fullerenes [1] have been widely investigated for possible applications in several fields, including superconductivity [2], non-linear optics [3], ferromagnetism [4] and medical field [5]. Furthermore, the interest in fullerenes has recently boosted due to the application in solar cells [6-8].

Fullerenes come in a large variety of structures, whose complexity grows with the size, due to the increasing number of structures that fulfil the "Isolated Pentagon Rule". This has stimulated an enormous computational effort to determine the energy lowest-lying structure of a given size [9]. In applications such as linear optics devices, higher fullerenes are considered to be more performing, than lower ones [10]. Furthermore, the use of higher fullerenes is an issue when chirality is involved [11].

On the experimental side, the structural characterization of higher fullerenes is hampered by the small quantities of fullerenes of a specific size as well as by the lengthy extraction operations. Fullerenes can be separated by high performance liquid chromatography (HPLC) on a number of stationary phases. This is remarkable if we consider the similarity of the carbon spheroids, which differ mainly in shape, and electronic properties of their $\pi$-systems, in addition to slight variations in size [12]. Therefore, although the existence of fullerene isomers up to C540 [12] has been hypothesized, only isomers up to $\mathrm{C} 116$ have been, so far, isolated [13] and only the structure of isomers up to C96 has been determined [14]. Derivatization is also an effective tool for isolating a specific isomer, since it enhances the variation of physical properties [15]. A way of circumventing the difficulties of lengthy and expensive fullerene purification steps is using the powder as it is obtained from the synthesis, provided it is properly characterized. To fulfill this aim, we purchased fullerite, a mixture of fullerenes. According to the provider's specifications, the synthesis was performed using the arc-synthesis method in helium, developed by W. Kratschmer, et al. [16], and collecting the carbon soot after the carbon-arc evaporation.

\section{Experimental Methods}

Raw fullerene soot known as "fullerite" was purchased from Sigma-Aldrich (USA). A part of the fullerite underwent an extraction procedure by toluene, where only C60 and C70 are soluble. All the other higher fullerenes are insoluble in toluene and remain trapped. The extraction of C60 and C70 from fullerite is not exhaustive and residual amounts of these two fullerenes may remain into the toluene-extracted soot. 
Energy Dispersive X-ray Dispersion (EDXD) measurements of the finely ground fullerenes were performed using a noncommercial energy scanning diffractometer [17]. White Bremsstrahlung radiation emitted by a tungsten tube $(50 \mathrm{kV}, 40 \mathrm{~mA})$ was used. The sample was loaded in a quartz capillary $(2 \mathrm{~mm}$ diameter, $80 \mathrm{~mm}$ length). Scattering intensities of the sample were obtained by merging several measurements performed in correspondence to a set of scattering angles $\theta=24.0,8.0^{\circ}, 3.0^{\circ}, 1.0^{\circ}, 0.5^{\circ}$. This allowed us to cover a wide range of the scattering variable $\mathrm{q}$, between 0.12 and $19.56 \AA^{-1}$. The expression for $\mathrm{q}$ is $\mathrm{q}=4 \pi \sin \theta / \lambda \approx 1.014 \mathrm{E} \sin \theta$ when $\mathrm{E}$ is expressed in $\mathrm{keV}$ and $\mathrm{q}$ in $\AA^{-1}$. The primary beam intensity $\mathrm{I}_{0}(\mathrm{E})$ was measured directly, by reducing the tube current to $10 \mathrm{~mA}$ without the sample. Transmission of the samples was measured under the same conditions. Both quantities are needed to carry out the necessary absorption corrections to experimental data [18]. The various angular data were normalized to a stoichiometric unit of volume containing one molecule and merged to yield the scattered intensity, $I_{\text {E.U. }}$ (q), expressed in electron units. Finally, the independent scattering was subtracted from I E.U. (q) to obtain the total static structure function i(q), also termed "reduced intensity", according to the relation

$$
i(q)=I_{E . U .}(q)-\sum x_{i} f_{i}^{2}
$$

In this equation, $x_{i}$ and $f_{i}$ are the number concentration and the scattering factor of carbon atoms, respectively. This function was multiplied by $\mathrm{q}$ and by a q-dependent sharpening factor, $\mathrm{M}(\mathrm{q})$, to improve the curve resolution at high q. The expression for M(q), using carbon as the "sharpening atom", is

$$
M(q)=\frac{f_{C}^{2}(0)}{f_{C}^{2}(q)} \exp \left(-0.01 \mathrm{q}^{2}\right)
$$

We approached the structural analysis of the carbon soot, by comparing the experimental X-ray scattered intensity qi(q) $\mathrm{M}(\mathrm{q})$ to the theoretical one of homogeneous fullerenes, i.e. fullerenes of a single size. We probed models of all sizes that were, to our knowledge, resolved, hence with available structural coordinates: C60 [19], C70 [20], C74 [21], C76 [22], C80 [23], C82 [24], C84 [25], C90 [26], C96 [27]. Furthermore, a simulation with the hypothesized structure of fullerene C180 [28] was made, using the cartesian coordinates published in the webpage http://www.nanotube.msu.edu/fullerene/fullerene-isomers.html.

Runs of Molecular Dynamic simulations of 100 or 800 homogeneous fullerenes randomly distributed in a box of finite dimensions, followed by calculation of the scattered intensity yielded the theoretical curves.

MD simulations were performed at ambient conditions using the AMBER package [29] with standard cubic periodic boundary conditions and the generalized amber force field (GAFF).

After the initial conjugate gradient minimization, the system was equilibrated for 500000 steps in NPT conditions (target temperature and pressure $1 \mathrm{~atm}$ and $300 \mathrm{~K}$, respectively). The equilibrium simulated density turns out to be $1.53 \mathrm{~mol} / \mathrm{dm}^{3}$, which is in good agreement with the reported experimental value of $1.59 \mathrm{~mol} / \mathrm{dm}^{3}$. A productive NVT simulation followed, using the same time step ( $2 \mathrm{fs})$; the last $1 \mathrm{~ns}$ was accumulated every $0.1 \mathrm{ps}$ for the evaluation of scattered intensity. The scattered intensities were obtained from the pair distribution functions [30], using a purposely written program.
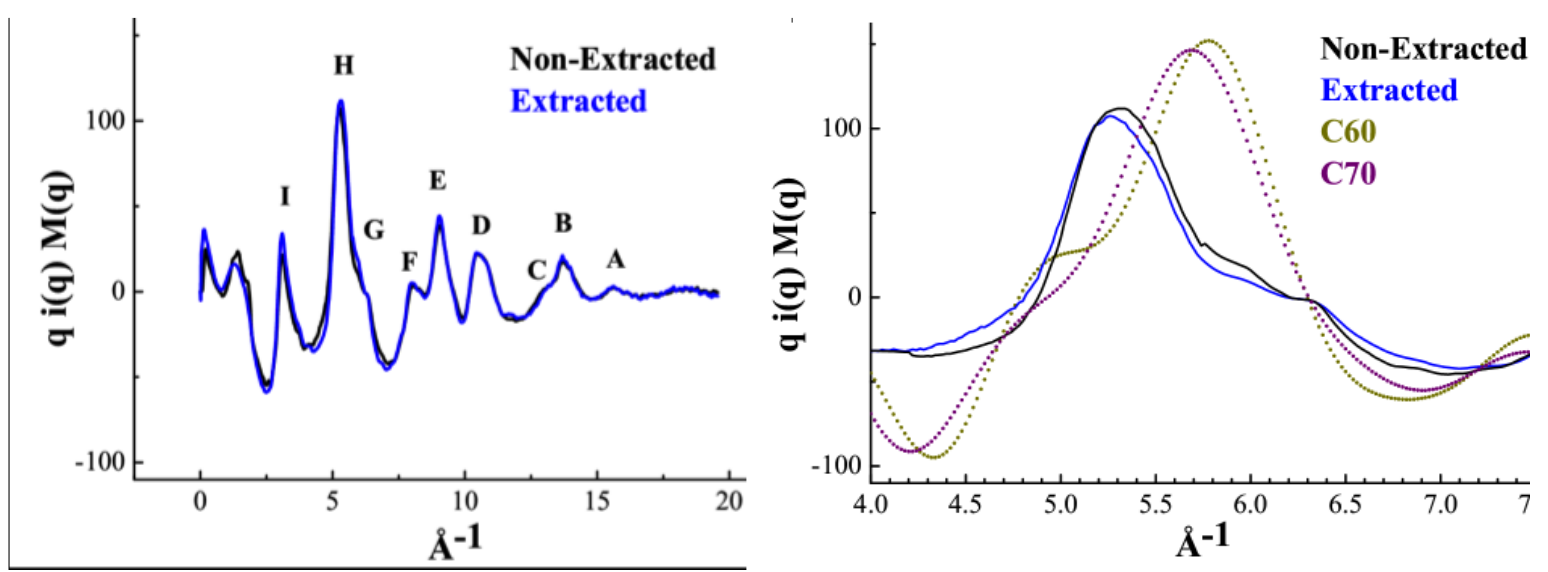
FIGURE 1. Left: Scattered Intensity of fullerite (black solid line) and fullerite upon extraction by toluene (blu solid line) as a function of the scattering parameter. Right: Scattered Intensity of non-extracted and extracted fullerite in the q range 4-7.5 $\AA-1$. For comparison theoretical scattered intensities of C60 (green dots) and C70 (purple dots) are reported.

\section{Results and Discussion}

The analysis of the experimental curve, i.e. the building up of the theoretical peak shapes to compare to, was carried on by molecular dynamics modeling of the interactions between spheroids, in order to determine the majority component of the mixture. Furthermore, a finer tuning of the overall experimental structure was performed by calculating a weighted average of the "single" structures.

None of the homogeneous fullerene curves overlaps completely with the experimental one. If we consider only the resolved structures of single fullerenes, the agreement improves with the fullerene size and it is higher with the C96 model. An even better agreement is achieved with the hypothesized C180. The weighted average of the theoretical curves of homogeneous fullerenes yields a tentative composition of the soot. However, the standard deviation is very high, pointing at the presence of fullerenes higher than $\mathrm{C} 180$ in the soot, whose contribution has not been considered; amorphous fractions could be present as well. Tests on the box size of the MD simulations (100 vs 800 molecules) do not show significant differences.

Scattered intensity was collected from the soot as well as from a portion of the fullerite extracted with toluene. This procedure generally removes C60 and C70 fullerenes, though not quantitatively, from the soot and leaves all higher fullerenes. Both spectra are reported on the left hand part of Fig. 1. The two spectra show small differences that are evidenced in the right-hand side of the figure, in the q range 4-7.5 $\AA^{-1}$. In this plot also the theoretical intensities of C60 and C70 are reported as comparison. In this range, for instance the peak at $5.24 \AA^{-1}$ as well as the shoulder around $6 \AA^{-1}$ are slightly shifted towards lower values upon extraction. This can be related to the partial suppression of the contribution of the peaks at 5.8/5.9 $\AA^{-1}$ of the low fullerenes.

The overall structure function curve can be ideally divided into two zones, the first one between $0-7 \AA^{-1}$ and the second one between 7-17 $\AA^{-1}$. Though all interactions between all atoms contribute to the scattered intensity, the weight of each contribution is different in different portions of the curve. This allows a rough distinction of the curve into two parts: the first one, where the intermolecular interactions prevail and the second one, where the intramolecular interactions are predominant. This distinction allows a preliminary consideration, i.e. the peaks intensity in the lower q region is rather low, hinting that the distance between fullerene particles tends to be small, mostly in the same range as the inner bond distances.

In first instance, we analyze the second portion of the experimental curve (intramolecular distances, peaks A through F) and we evaluate the agreement between curves in terms of peak position and peak area. The comparison of experimental scattered intensity in the larger q region with the theoretical intensity of the different models shows a generally improving agreement with the increasing fullerene size (all experimental and model patterns are reported in Fig. 2). In the C60 model, there is hardly any overlap between experimental and theoretical curve. In the C70 model, three of the features, i.e. $\mathrm{A}, \mathrm{B}$ and $\mathrm{C}$ are peaked at the correct $\mathrm{q}$ value, though feature $\mathrm{B}$ is slightly overestimated. The other features are slightly misplaced and/or of the wrong intensity. In the C74 model the agreement improves: peaks $\mathrm{A}, \mathrm{B}$ and $\mathrm{C}$ are well reproduced and also peak $\mathrm{D}$ is at the correct position, though overestimated. The agreement between theoretical and experimental curve improves further in the $\mathrm{C} 76$ model. Peaks $\mathrm{A}, \mathrm{B}, \mathrm{C}$ and $\mathrm{D}$ are well reproduced, with a small underestimation of peak B.

The theoretical model of $\mathrm{C} 80$ has more wiggles and hardly reproduces any intensity of the experimental curve. Fullerenes models of larger size reproduce to some extent also peaks at lower q values. In particular, in models C82 and $\mathrm{C} 84$, the position of all peaks A through $\mathrm{E}$ is matched. As for the intensities, in both cases, only peak $\mathrm{D}$ is higher than in the experimental curve.

C90 and above all C96 (Fig. 3, left) are definitively the models best matching the experimental curve. In the C90 model also the peak $\mathrm{F}$ at $10.56 \AA^{-1}$ is partly reproduced, (the trough at $9.92 \AA^{-1}$ between peaks $\mathrm{E}$ and $\mathrm{F}$, however, is still not matched). The $\mathrm{C} 96$ model reproduces fairly well the curve between 9.92 and $20.00 \AA^{-1}$. Furthermore, also the peak at $9.02 \AA^{-1}$ is rather well reproduced. 

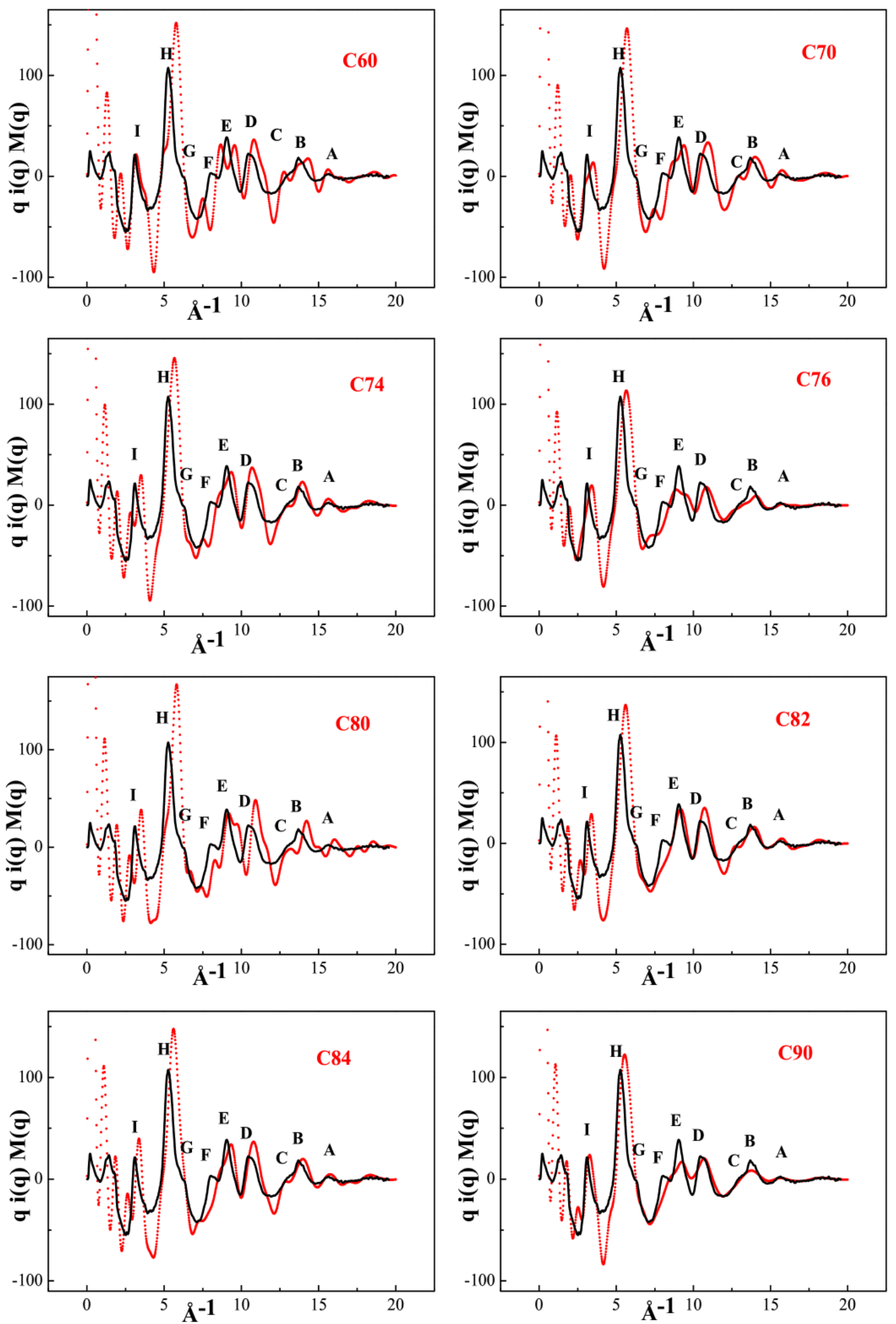

FIGURE 2. Experimental Scattered Intensity (black solid line) and Theoretical Scattered Intensity (red dotted line) of the simulated fullerenes: C60, C70, C74, C76, C80, C82, C84, C90. 

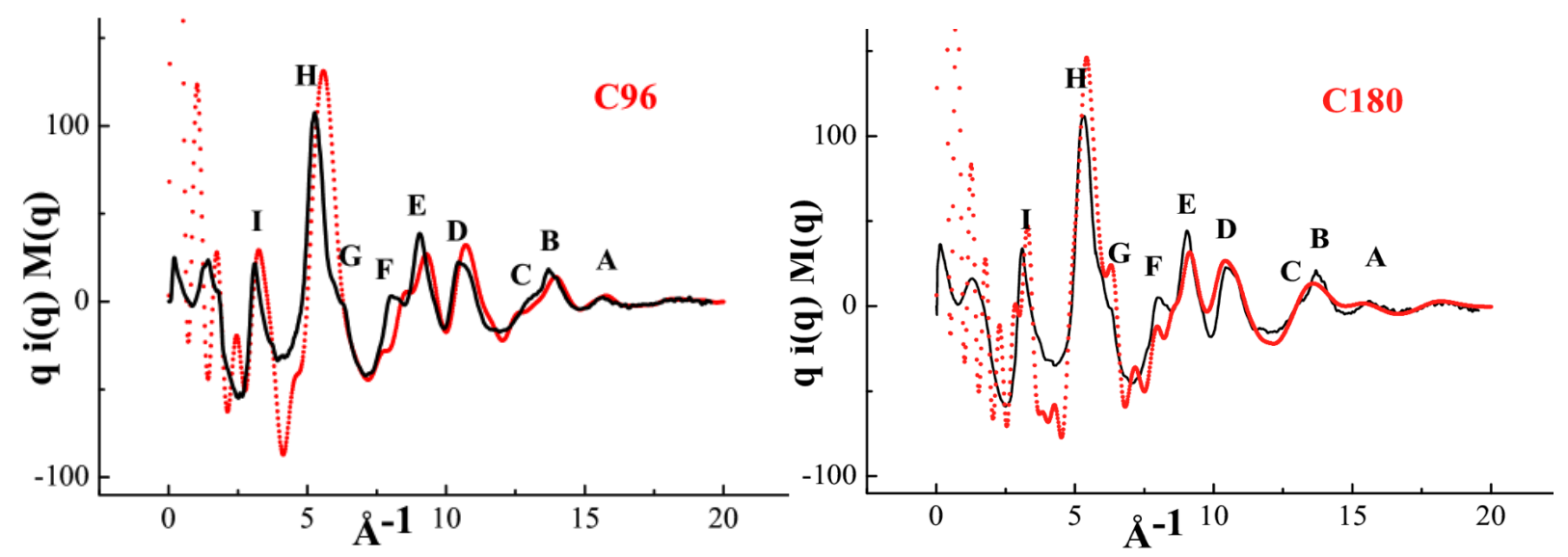

FIGURE 3. Experimental Scattered Intensity (black solid line) and Theoretical Scattered Intensity (red dotted line) of C96 (crystal structure, left) and C180 (hypothesized, right), that give the best agreement among all the structures considered.

The first part of the curve, mostly related to the inter-molecular interactions is not completely matched in any of the models. However, the discrepancies are larger for the C60 and C80, where also the intra-molecular distances are badly reproduced. Also for the portion of the curve at lower q values, the best correspondence is obtained with the C90 and C96 models especially for peaks H and I. Peak G in not fitted in any of the experimentally resolved models.

If we consider C180 (Fig. 3, right), whose structure has only been hypothesized, we find that there is a good match with the experimental curve, even in comparison with C96. In particular, features $\mathrm{A}, \mathrm{B}, \mathrm{C}, \mathrm{D}$ and $\mathrm{E}$ are rather well reproduced. Also peak F, finally appears in a theoretical structure, as well as the feature G, though a little too pronounced. Peaks $\mathrm{H}$ and I are slightly overestimated.

Being fullerite a mixture several different sized fullerenes, the overall diffraction pattern can be seen as the sum of the single curves scattered intensity. The contribution of each fullerene and, as a consequence, the percentage of each fullerene in the soot, can be evaluated through a weighted average the theoretical curves where the weighting parameters are tuned against the experimental curve. The fitting curve is $\mathrm{y}=(\mathrm{aC} 60+\mathrm{bC} 70+\mathrm{cC} 74+\mathrm{dC} 76+\mathrm{eC} 80+\mathrm{fC} 82+\mathrm{gC} 84+\mathrm{hC} 90+\mathrm{iC} 96) / 9$ and coefficients of the best fit are reported in Table 1 .

TABLE 1. Weight coefficients of the fullerenes contribution to the total theoretical curve

\begin{tabular}{cc}
\hline Coefficient & Value \\
\hline a $(\mathrm{C} 60)$ & 0.795819 \\
$\mathrm{~b}(\mathrm{C} 70)$ & 0.090993 \\
$\mathrm{c}(\mathrm{C} 74)$ & 0.002028 \\
$\mathrm{~d}(\mathrm{C} 76)$ & 0.037475 \\
$\mathrm{e}(\mathrm{C} 80)$ & 0.012276 \\
$\mathrm{f}(\mathrm{C} 82)$ & 0.005132 \\
$\mathrm{~g}(\mathrm{C} 84)$ & 0.009006 \\
$\mathrm{~h}(\mathrm{C} 90)$ & 0.010619 \\
$\mathrm{i}(\mathrm{C} 96)$ & 0.036652 \\
\hline
\end{tabular}

The fit was performed by in the range $2.5-20 \AA^{-1}$, i.e. excluding the first part of the curve, where the theoretical simulations fail, owing to the likely too small dimensions of the simulation boxes, the lack of three-body terms in the simple potentials, etc. The resulting averaged theoretical curve is reported in Fig. 3 in comparison with the experimental one. The standard deviation is definitively high: 13.01. The coefficients of the theoretical curve show a sort of homogeneous composition of the soot, with all "known" fullerenes present at various extents. However, the fit of the theoretical mixed curve towards the experimental one is rather poor and no clear-cut conclusion can be really drawn on the exact composition. A complete fit of the curve could be obtained by including, among the theoretical curves, also those belonging to even higher fullerenes, whose existence and exact structure has, so far, 
only been hypothesized. We think, therefore, that fullerenes higher than C96 exist and contribute to the total scattered intensity of the soot. The resolution of those exact structures will allow EDXD to discriminate the soot composition. The effect of larger simulation boxes was evaluated for C96, but the theoretical curves do not seem to be very effected.

In summary, we have shown that EDXD measurements can be successfully employed to analyze a commercial fullerite sample of unknown composition. This feature confirms and extends the applicability of the technique to the field of Materials Science, already demonstrated in several studies [31]. Data analysis was carried in terms of single homogeneous fullerene contribution. The complete scattered intensities of C60, C70, C74, C76, C80, C82, C84, C90, C96 and C180 (the structure of which is only hypothesized) was calculated by sampling snapshots of a molecular dynamics trajectory. The agreement between each single theoretical curve and the experimental one has been evaluated and shows a better agreement with C96, among the experimentally resolved structures, and with the hypothetical C180. A weighted average of the theoretical curves has been made and the weighting parameters tuned against the experimental curve. The minimum standard deviation has been achieved with a contribution of all curves to various extents. However, the agreement is rather poor. We find it a likely scenario that fullerenes higher than C96 contribute to the total experimental curve. Their isolation and the determination of their structure will allow

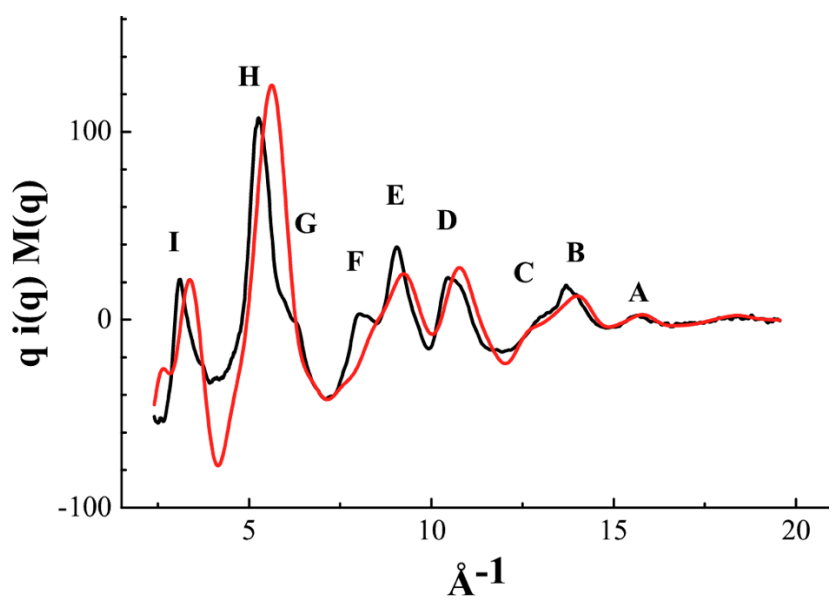

FIGURE 4. Experimental Scattered Intensity (black solid line) and Theoretical Scattered Intensity (red solid line) calculated as weighted average of the single homogeneous fullerenes

finer analysis of the fullerite soot by EDXD as well.

\section{ACKNOWLEDGMENTS}

The authors wish to thank Prof. Ruggero Caminiti for providing EDXD spectra and for general guide and assistance and Dr. Franco Cataldo for providing the sample. Computational support from CASPUR (grant std11465, std12-011) is acknowledged; L. G. acknowledges support from FIRB (RBFR086BOQ)

\section{REFERENCES}

1 H. W. Kroto, J. R. Health, S. C. Obrien, R. F. Curl, and R. E. Smiley, Nature (London) 318, 162 (1985).

2 H. Takeya, K. Miyazawa, R. Kato, T. Wakahara, T. Ozaki, H. Okazaki, T. Yamaguchi and Y. Takano, Molecules 17(5), 4851 (2012).

3 E. F. Sheka, Nanosci. Nanotechnol. Lett., 3(1), 34 (2011).

4 S. Ren and M. Wuttig, Adv. Mater. 24(6), 724 (2012).

5 R. Bakrym, R. M. Vallant, M. Najam-ul-Haq, M. Rainer, Z. Szabo, C. W. Huck and G. K. Bonn, Int J Nanomedicine 2(4), 639 (2007).

6 S. Wilken, V. Wilken, J. Parisi, and H. Borchert, Organic Electronics: physics, materials, applications 13(11), 2386 (2012)

7 M. Anafcheh, R. Ghafouri and N. L. Hadipour, Solar Energy Materials and Solar Cells 105, 125 (2012).

8 C.-Z. Li, H.-L. Yip and A.K.-Y Jen, J. Mater. Chem. 22(10), 4161 (2012)

9 N. Shao, Y. Gao, S. Yoo, W. An and X. C. Zeng, J. Phys. Chem. A, 110, 7672 (2006) 
10 K. Harigaya, J. Luminescence 76\&77, 652 (1998).

11 C. Tilghen and F. Diederich, Chem. Rev. 106, 5049 (2006).

12 P. Calaminici, J. Carmona-Espindola, G. Geudtner, and A. M. Köster, Int. J. Quantum Chem. 112(19) 3252 (2012).

13 H. Richter, A. J. Labrocca, W. J. Grieco, K. Taghizadeh, A. L. Lafleur and J. B. Howard, J. Phys. Chem. B 101, 1556 (1997).

14 H. Yang, H. Jin,Y. Che, B. Hong, Z. Liu, J. A. Gharamaleki, M. M. Olmstead and A. L. Balch, Chem. Eur. J. 18, 2792 (2012).

15 N. B. Tamm, Lev N. Sidorov, E. Kemnitz and S. I. Troyanov, Angew. Chem. Int. Ed. 48, 9102 (2009)

16 W. Kratschmer, K. Fostiropoulos and D. R. Huffman, Chem. Phys. Lett. 170, 167 (1990).

17 R. Caminiti, M. Carbone, S. Panero and C. Sadun, J. Phys. Chem B. 103(47), 10348 (1999); M. Carbone, R. Caminiti and C. Sadun, J. Mater. Chem. 6, 1709 (1996); V. Migliorati, P. Ballirano, L. Gontrani, A. Triolo and R. Caminiti, J. Phys. Chem. B 115(17), 4887 (2011).

18 K. Nishikawa and T. Ijima, Bull. Chem. Soc. Jpn. 57, 1750 (1984); V. Rossi Albertini, L. Bencivenni, R. Caminiti, F. Cilloco, C. Sadun, J. of Macromolecular Science, Part B: Physics 35(2), 199 (1996).

19 M. V. Korobov, A. L. Mirakian, N. V. Avramenko, E. F. Valeev, I. S. Neretin, Y. L. Slovokhotov, A. L. Smith, G. Oloffson and R. S. Ruoff, J. Phys. Chem. B 102, 3712 (1998)

20 M. Schulz-Dobrick, M. Panthofer and M. Jansen, Cryst. Eng. Comm. 8, 163 (2006)

21 K. Friese, M. Panthofer, G. Wu and M. Jansen, Acta Crystallogr. Sect.B: Struct. Sci. 60, 520 (2004)

22 M.V. Korobov, A. L. Mirakian, N.V. Avramenko, E. F. Valeev, I. S. Neretin, Y. L. Slovokhotov, A. L. Smith, G. Oloffson and R. S. Ruoff, J. Phys. Chem. B 102, 3712 (1998)

23 E.Nishibori, I. Terauchi, M. Sakata, M. Takata, Y. Ito, T. Sugai, H. Shinohara, J. Phys. Chem. B 110, 19215 (2006)

24 H. Kawada, Y. Fuji, H. Makao, Y. Murakami, H. Suematan, K. Kikuchi, Y. Achiba and I. Ikemoto, Phys. Rev. B 51, 8723 (1995)

25 L. Epple, K. Amsharov, K. Simeonov, I. Dix and M. Jansen, Cryst. Commun. 5610 (2008)

26 H. Yang, B.Q. Mercado, H. Jin, Z. Wang, A. Jiang, Z. Liu, C. M. Beavers, M. M. Olmstead and A. L. Balch, Chem. Commun. 47, 2068 (2011)

27 H. Yang, H. Jin, Y. Che, B. Hong, Z. Liu, J. A. Gharamaleki, M. M. Olmstead, and A. L. Balch, Chem. Eur. J. 18, 2792 (2012)

28 See, for instance, http://www.nanotube.msu.edu/fullerene/fullerene-isomers.html

29 D.A. Case, T.A. Darden, T.E. Cheatham, III, C.L. Simmerling, J. Wang, R.E. Duke, R. Luo, R.C. Walker, W. Zhang, K.M. Merz, B. Roberts, S. Hayik, A. Roitberg, G. Seabra, J. Swails, A.W. Goetz, I. Kolossvai, K.F. Wong, F. Paesani, J. Vanicek, R.M. Wolf, J. Liu, X. Wu, S.R. Brozell, T. Steinbrecher, H. Gohlke, Q. Cai, X. Ye, J. Wang, M.-J. Hsieh, G. Cui, D.R. Roe, D.H. Mathews, M.G. Seetin, R. Salomon-Ferrer, C. Sagui, V. Babin, T. Luchko, S. Gusarov, A. Kovalenko, and P.A. Kollman (2012), AMBER 12, University of California, San Francisco.

30 C. J. Pings and J. Waser, J. Chem. Phys. 48, 3016 (1968)

31 a) A. Capobianchi, G. Pennesi, A. M. Paoletti, G. Rossi, R. Caminiti and C Sadun, Inorg. Chem. 35 (16), 4643 (1996); b) R Caminiti, M. Gleria, K.B. Lipkowitz, G.M. Lombardo and G.C. Pappalardo J. Am. Chem. Soc. 119 (9), $2196-2204$ (1997); c) L. Abis, D.Belli Dell'Amico, C. Busetto, F. Calderazzo, R. Caminiti, C. Ciofi, F. Garbassi and G. Masciarelli, J. Mater. Chem. 8 (3), 751-759 (1998) 